\title{
Rapid and Label-Free Screening of Enzyme Inhibitors Using Segmented Flow Electrospray Ionization Mass Spectrometry
}

\author{
Jian Pei, ${ }^{\text {a }}$ Qiang Li, ${ }^{a}$ and Robert T. Kennedy ${ }^{a, b}$ \\ a Department of Chemistry, University of Michigan, Ann Arbor, Michigan, USA \\ ${ }^{\mathrm{b}}$ Department of Pharmacology, University of Michigan, Ann Arbor, Michigan, USA
}

Electrospray ionization mass spectrometry (ESI-MS) is an attractive analytical tool for high-throughput screening because of its rapid scan time and ability to detect compounds without need for labels. Impediments to the use of ESI-MS for screening have been the relatively large sample consumed and slow sample introduction rates associated with commonly used flow injection analysis. We have previously shown that by segmenting nanoliter plugs of sample with air, an array of discrete samples can be delivered to a platinum-coated emitter tip for ESI-MS analysis with throughput as high as $0.8 \mathrm{~Hz}$ and carry-over between samples less than $0.1 \%$. This method was applied to screening for inhibitors of acetylcholinesterase as a demonstration of the potential of segmented flow ESI-MS for such applications. Each enzyme assay consumed $10 \mathrm{~nL}$ of sample. At $1 \mu \mathrm{L} / \mathrm{min}$ infusion rate, 102 samples were analyzed, corresponding to a $0.65 \mathrm{~Hz}$ sample analysis rate. Linear quantification of choline was achieved from $200 \mu \mathrm{M}$ to $10 \mathrm{mM}$ using this method and $Z^{\prime}$ values were over 0.8 for the assay. Detailed pharmacologic dose-response curves of selected inhibitors were also measured in high-throughput fashion to validate the method. (J Am Soc Mass Spectrom 2010, 21, 1107-1113) (C) 2010 Published by Elsevier Inc. on behalf of American Society for Mass Spectrometry

$\mathrm{D}$ rug discovery often requires identification of lead compounds from combinatorial libraries containing millions of candidates. High-throughput screening (HTS) is necessary for such large scale sample handling and measurement [1]. In vitro biochemical assay in multiwell plates with optical detection has been the primary format for HTS [2-4]. A drawback of optical detection is that usually either labels or indicator reactions must be incorporated into the assay to generate detectable signal. This requirement results in several problems, including increased difficulty of assay development, increased cost because of added or complex reagents, and greater potential for inaccurate results if test compounds affect the label or indicator reaction rather than the test reaction. High-throughput assays that can be performed without labels or indicator reactions are therefore of great interest [5].

A potentially powerful label-free detection system is electrospray ionization mass spectrometry (ESI-MS). Indeed, a variety of ESI-MS assays for enzymes and noncovalent biomolecule binding have been developed and explored for screening applications [5-7]. The throughput achievable by ESI-MS is limited by the need to interface the mass spectrometer to multiwell plates and perform individual injections for each assay (this

Address reprint requests to Dr. R. T. Kennedy, Department of Chemistry, University of Michigan, Ann Arbor, MI 48109, USA. E-mail: rtkenn@ umich.edu limit assumes the standard procedure of testing one compound at a time; for certain assays, MS can analyze a mixture of test compounds at one time $[8,9])$. Currently, individual samples are most often introduced to a mass spectrometer by flow injection, i.e., loading sample into an HPLC-style injection valve and then pumping it through the ESI emitter. It is a significant challenge to engineer a rapid injection system that uses small volumes, has low carry-over between injections, uses low flow rates, and is reliable. A rapid system that requires just 4 to $5 \mathrm{~s}$ per analysis and consumes 1 to $5 \mu \mathrm{L}$ of sample has been commercialized [10]; however, more common systems are considerably slower and require a few minutes per sample. For HTS, it is desirable to lower the volume of sample consumed, to reduce reagent costs, and further increase throughput. A potentially interesting alternative to flow injection is the use of microfabricated nanospray emitter arrays that can be interfaced to multiwell plates using a liquid handling robot (e.g., NanoMate by Advion BioSciences Inc., Ithaca, NY, USA). This system uses a few microliters to load nanospray tips and a cycle time of $40 \mathrm{~s} /$ sample [11].

In this work, we eliminated the need for flow injection by utilizing segmented flow analysis for highthroughput ESI-MS. Segmented flow has long been a popular method for high-throughput analysis in clinical settings [12]. In the classic scheme, individual samples are segmented by air in a tube, reagents added for colorimetric assay, and the samples passed through an 
optical detector $[12,13]$. The last few years have seen a resurgence of interest in segmented flow with the advent of sophisticated microfluidics that allow miniaturization (femtoliter to nanoliter samples) and new methods for manipulating sample plugs and droplets [14-16]. We have recently demonstrated the feasibility of directly pumping segmented flow through an ESI emitter tip to obtain mass spectrometric analysis of discrete sample plugs at high-throughput $(0.8 \mathrm{~Hz}$ analysis rate) with low carry-over [17]. Other groups have also reported ESI-MS of droplet samples [18, 19]. The success of these methods suggests that they may be applicable to rapid, label-free screening of assay mixtures.

As a test system, we demonstrate screening for inhibitors of acetylcholinesterase (AchE). AchE catalyzes conversion of acetylcholine (Ach) to choline and is the primary way of terminating Ach signaling at synapses. Inhibition of AchE is a possible treatment for Alzheimer's disease (AD) and related dementia [20, 21]. While a handful of AchE inhibitors have been approved for AD treatment, searching for compounds with improved pharmacologic and toxicologic properties remains an active pursuit [22].

Because the AchE reaction does not generate components that are easily detected optically, screening has required coupling the enzyme with indicator reactions [23-25]. It has been demonstrated that AchE assays can be performed using flow-injection ESI-MS [26] and HPLC-MS [27] to directly detect substrate and/or product of the reaction. Throughput of $0.2 \mathrm{~Hz}$ with 1 to $5 \mu \mathrm{L}$ of sample consumption was possible when using an automated sampling and injection system [27]. In this work, we demonstrate that with direct ESI-MS analysis of segmented assay mixtures, we can generate a throughput of $0.65 \mathrm{~Hz}$ for AchE inhibitor screening while consuming $10 \mathrm{~nL}$ of sample. Excellent reproducibility indicated by $\mathrm{Z}^{\prime}$ values above 0.8 was also obtained.

\section{Experimental}

\section{Chemicals and Reagents}

Water and methanol were purchased from Burdick and Jackson (Muskegon, MI, USA). Acetic acid was purchased from Fisher Scientific (Pittsburgh, PA, USA). All other chemicals were obtained from Sigma (St. Louis, MO, USA).

AchE activity measurement. Assay conditions were modified from a previously reported method [28]. Ten $\mathrm{mM} \mathrm{NH} \mathrm{HCO}_{3}$ was used as reaction buffer for all AchE experiments. AchE (from Electrophorus electricus, type VI-S) was prepared daily from lyophilized powder at 90 $\mu \mathrm{g} / \mathrm{mL}$ solution; $2 \mu \mathrm{L}$ solution of drug to be tested was mixed with $20 \mu \mathrm{L}$ AchE solution and incubated on ice for $30 \mathrm{~min}$ before being brought to room-temperature; $20 \mu \mathrm{L} 200 \mathrm{mM}$ acetylcholine iodide solution was then added to above AchE solution to start hydrolysis. After
20 min incubation, $180 \mu \mathrm{L}$ of an ice-cold mixture containing $1 \mathrm{mM}$ chlormequat, $60 \%$ (vol/vol) methanol, and $1.5 \%(\mathrm{vol} / \mathrm{vol})$ acetic acid was rapidly mixed with $20 \mu \mathrm{L}$ of the enzyme mixture to terminate the reaction; $30 \mu \mathrm{L}$ of each final quenched reaction mixtures were pipetted into a 384-well plate (Corning, Fisher Scientific, Pittsburg, PA, USA) for loading into a sample tube for analysis.

Sample plug generation. Air-segmented sample plugs were generated using the system illustrated in Figure 1, similar to that recently described [17]. A Teflon tube of $75 \mu \mathrm{m}$ i.d. and $360 \mu \mathrm{m}$ o.d. (IDEX Health and Science, Oak Harbor, WA, USA) was used for sampling and storing sample plugs. One end of this tubing was connected to a $100 \mu \mathrm{L}$ syringe (Hamilton; Fisher Scientific, Pittsburg, PA, USA) using a $250 \mu \mathrm{m}$ bore PEEK union (Valco Instruments, Houston, TX, USA). The syringe and Teflon tubing were initially filled with Fluorinert FC-40 (Sigma). The syringe was mounted on a PHD 200 programmable syringe pump (Harvard Apparatus, Holliston, MA, USA). To fill the tube with air-segmented samples, a computer-controlled xyzmicropositioner (built in-house from XSlide assemblies; Velmex Inc., Bloomfield, NY, USA) was used to move the inlet of the Teflon tubing from sample-to-sample on the multiwell plate while the pump was operated at a fixed aspiration rate. By using an aspiration rate of 200 $\mathrm{nL} / \mathrm{min}, 10 \mathrm{~nL}$ sample plugs and $4 \mathrm{~mm}$ long air plugs were produced. Using this procedure, a tube could be filled with 100 samples in $\sim 10 \mathrm{~min}$. The relative standard deviation of sample plug size was $25 \%$ due to the compressibility of air affecting the sampling rate with increasing amount of air aspired into the tube.

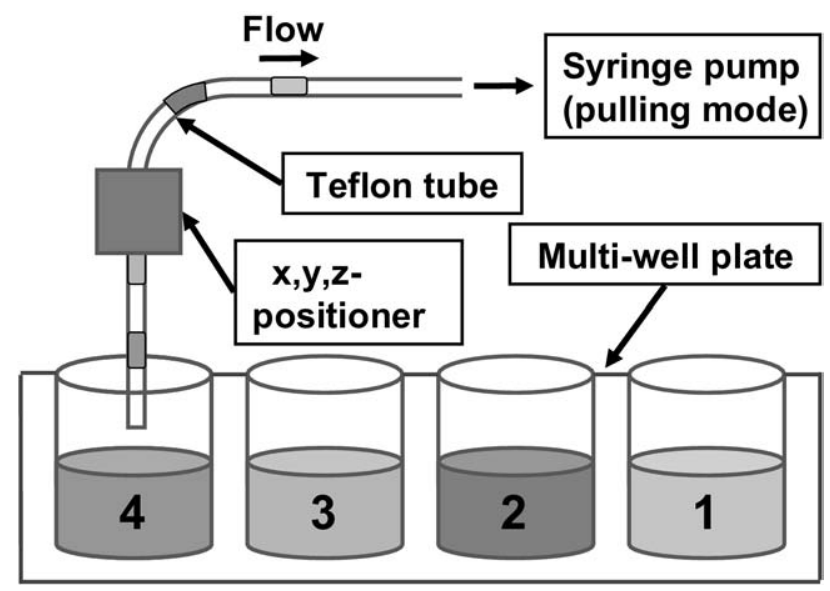

Figure 1. Diagram of system for generating air-segmented sample plugs from a multiwell plate. Cartridges of sample plugs were prepared by dipping the tip of a $75 \mu \mathrm{m}$ i.d. Teflon tubing prefilled with Fluorinert FC-40 into sample solution stored in a multiwell plate, aspirating a desired volume, retrieving the tube, aspirating a desired volume of air, and moving to the next well until all samples were loaded. Movement of the tubing was controlled with an automated micropositioner and sample flow was controlled with a syringe pump connected to the opposite end of the tubing. 
ESI-MS and data analysis. After sample plug generation, the inlet end of the Teflon tubing was connected to a Pt-coated fused-silica electrospray emitter (FS 360-508-CE; New Objective, Woburn, MA, USA), which was $50 \mu \mathrm{m}$ i.d. and pulled to $8 \mu \mathrm{m}$ i.d. at the tip, using a 360 i.d. Teflon connector. The emitter was mounted in a nanospray source (PV-550; New Objective). A syringe pump operated at $1.0 \mu \mathrm{L} / \mathrm{min}$ was used to drive sample plugs through the emitter poised at $+1.7 \mathrm{kV}$ for ESI-MS analysis. The MS used was a LTQ XL linear ion trap MS (Thermo Fisher Scientific, Waltham, MA, USA) operated in single-stage, full-scan mode with following settings: automatic gain control (AGC) on, negative mode, $50-300 \mathrm{~m} / \mathrm{z}$ scan range, and micro scan number $=$ 1. Scan time was $\sim 0.1 \mathrm{~s}$. Reconstructed ion current traces (RIC) of choline $(\mathrm{m} / \mathrm{z}$ 104) and chlormequat $(\mathrm{m} / \mathrm{z}$ 122) were extracted from total ion current (TIC). Peak marking and analysis were performed automatically by Qual Browser. For determining inhibitor $\mathrm{IC}_{50}$ values, GraphPad Prism 3.0 (GraphPad Software, San Diego, CA, USA) was used for the curve fitting.

\section{Results and Discussion}

\section{AchE Assay}

Initial experiments were directed at determining AchE assay conditions that would be compatible with ESIMS. Incubating acetylcholine with AchE in $10 \mathrm{mM}$ $\mathrm{NH}_{4} \mathrm{HCO}_{3}$ buffer for $20 \mathrm{~min}$ at room temperature followed by quenching of the reaction by addition of a methanol and acetic acid mixture was found to be suitable. With this incubation time, $<10 \%$ of the original acetylcholine was consumed, thus ensuring linear hydrolysis rates. The quenching solvent was found to completely stop the enzymatic reaction and be compatible with MS. $\mathrm{NH}_{4} \mathrm{HCO}_{3}$ provided adequate buffering while being compatible with ESI and providing low ion suppression. To improve quantification, chlormequat was included in the quenching solution to act as an internal standard [28]. Typical MS spectra illustrating detection of substrate (acetylcholine), product (choline), and internal standard are shown in Figure 2. Under the electrospray conditions used, the spectra are free from interfering peaks from the Fluorinert FC-40 used for coating the Teflon tubing. Inhibitors added to the assay reduced the choline signal as shown by Figure 2 .

\section{Segmented Flow ESI-MS Analysis for Rapid Screening}

To demonstrate rapid screening of AchE inhibitors, a set of 32 compounds, including four known AchE inhibitors and 28 randomly picked compounds, were tested at $100 \mu \mathrm{M}$ each in the AchE assay mixtures. This concentration was chosen because preliminary experiments suggested that it allowed weaker inhibitors, such as malathion, to be detected. For screening, each compound was tested in triplicate, resulting in a total of 102

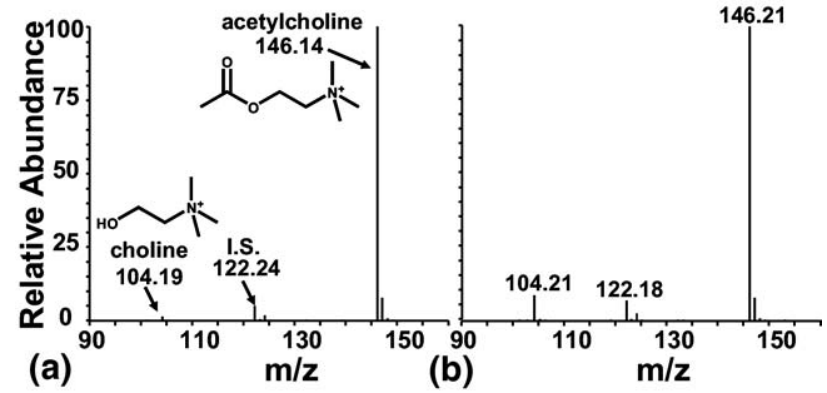

Figure 2. ESI mass spectra of quenched AchE assay mixtures after incubating $100 \mathrm{mM}$ acetylcholine, chlormequat (internal standard or I.S.), and $45 \mu \mathrm{g} / \mathrm{mL}$ AchE with (a) or without (b) 100 $\mu \mathrm{M}$ the AchE inhibitor neostigmine at room temperature for 20 min. AchE inhibition is detected by decrease of choline signal relative to control without inhibitor.

samples (96 assay samples, plus 3 blanks with no enzyme added, and 3 controls with no test compound added). These samples were loaded into a Teflon tube as a linear array using the procedure described in the Experimental section. Throughput of analysis is determined by sample plug volume and flow rate into the ESI source so that small sample volumes and high flow rates generate higher throughput. For this work, $10 \mathrm{~nL}$ sample plugs with $17 \mathrm{~nL}$ air gaps (or $4 \mathrm{~mm}$ spacing in a $150 \mu \mathrm{m}$ i.d. tubing) were chosen as a small-volume that was convenient to produce. Samples were pumped through the emitter at $1 \mu \mathrm{L} / \mathrm{min}$, which was the highest flow rate that did not cause the samples to merge in the emitter tip because of compression of the air segment.

These conditions allowed the 102 samples to be analyzed in $2.6 \mathrm{~min}$, corresponding to an analysis rate of $0.65 \mathrm{~Hz}$, as illustrated by ion current traces for the analysis shown in Figure 3a. Each sample is detected as a current burst followed by a period of zero current corresponding to the air segment passing through the emitter. As shown, the current rapidly stabilizes for each sample and remains steady as the sample is passed through the emitter. The presence of inhibitors is easily visualized by the reduced choline signal relative to internal standard signal in these traces. The inconsequential carry-over between samples is illustrated by the immediate step change in signal between samples of different choline concentrations.

The analysis rate of the segmented flow method compares favorably with previously reported flow injection AchE assays [26, 27, 29]. The speed of flow injection methods was limited by the need to inject individual samples or additional separation steps when assay buffer was not directly compatible with ESI-MS. It also seems likely to be able to further increase the analysis rate of the plug samples. Higher flow rates during analysis may be possible by using fluorocarbon oil to segment samples to avoid the effect of air compressibility. In preliminary studies with this approach, we have improved analysis rate to $\sim 2.3 \mathrm{~Hz}$. Also, generating lower volume samples by using smaller i.d. 

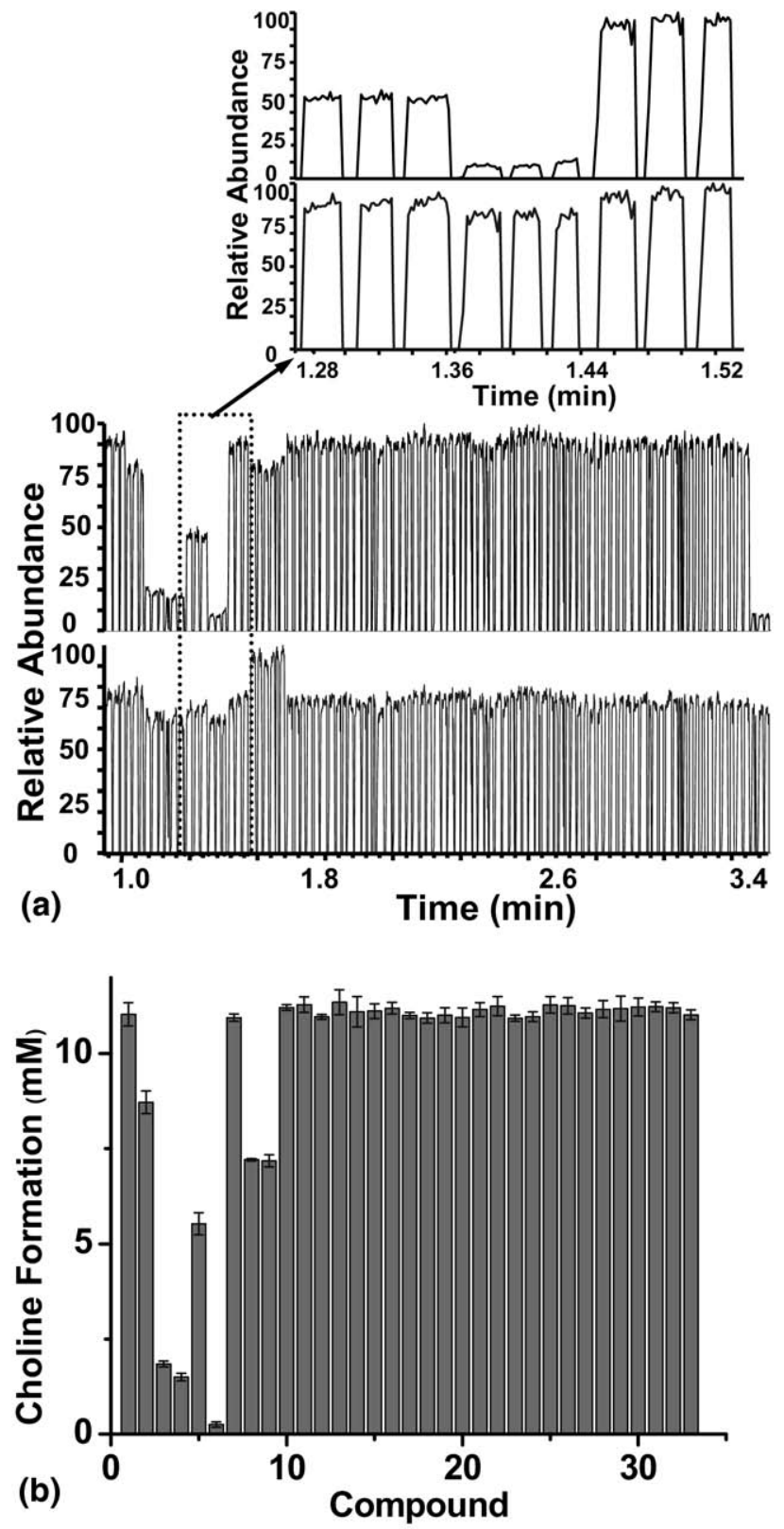

Figure 3. Screening of AchE inhibitors by segmented flow-ESIMS. (a) RIC trace for choline (top) and chlormequat (bottom) of 102 AchE enzyme assay sample plugs analyzed by ESI-MS in $2.6 \mathrm{~min}$. The series of samples tested 32 compounds in triplicate for AchE inhibition plus two control samples, also in triplicate. Compounds tested were, from left to right, control 1 (no drug added); malathion, neostigmine, eserine, edrophonium, isoproterenol, yohimbine, UK14,304, DMSO, serine, adenosine, thyronine, GABA, phenylalanine, alanine, proline, arginine, cysteine, lysine, tyrosine, glycine, arginine, glutamine, methionine, leucine, tryptophan, isoleucine, histidine, glutamic acid, aspartic acid, taurine, dopamine, valine, control 2 (no enzyme added). Inset shows signal for two inhibitors (edrophonium and isoproterenol) and one inactive compound. (b) Quantification of choline formed in each sample determined by subtracting background formation of choline and comparing choline signal (ratioed to internal standard) to calibration curve. Bars show mean concentration from triplicate samples with \pm 1 SD as error bar. sample tubing or by using a more sophisticated positioner that can move faster from well-to-well (relatively slow response time of the positioners used here prevented shorter aspiration times that would generate smaller samples) would be able to decrease the time required to analyze each sample. Ultimately, the analysis rate could become limited by the scan time of the mass spectrometer used. For example, if plugs are analyzed at $2 \mathrm{~Hz}$ and 10 mass spectra are acquired per sample, a scan rate of $20 \mathrm{~Hz}$ is required, comparable to many time-of-flight mass analyzers.

\section{Overall Throughput}

Although the rate of analysis was higher than previously reported ESI methods, the overall throughput must be considered. For example, the above comparison neglects the time required to load sample plugs into the tube for segmented flow analysis. In this proof of concept study, overall throughput was actually limited by sample loading because a single tube was loaded at a rate that required $6 \mathrm{~s}$ per sample; however, it would be a simple matter for a true HTS to load multiple tubes in parallel using either multiple syringe pumps or a single syringe pump with multiple barrels. Therefore, sample loading is not expected to have a significant effect on overall throughput of the segmented flow method. A more complicated, but intriguing, possibility to improve throughput would be to perform the entire assay in plugs. Several tools for manipulating plugs have been published, including mixing with streams, reagent addition, and splitting [14, 30, 31]. Thus, it is possible to envision a system in which a chemical library is stored as a series of plugs that are then tested and assayed by MS and by-passing the transfer from multiwell plate to tubing. Such an approach would also further decrease reagent usage.

Another issue that can be expected to impact overall throughput is sample preparation. The segmented flow assay was designed to be directly compatible with ESI and therefore did not require solid-phase extraction to remove salts or concentrate sample. It may not always be possible to design assays that allow direct injection into an ESI source. Current flow injection systems can utilize in-line solid-phase extraction at high flow rates so their throughput is minimally impacted by this necessity [26]. Therefore, for the segmented flow approach to be as versatile as the flow injection approach for HTS, it is necessary to develop systems that allow compatible solid-phase extraction or similar sample preparation steps that are high-throughput. One promising approach is parallel solid-phase extraction based on multiple fibers [32].

\section{Quantification}

To quantify choline production in the enzyme reaction, four different measurements were evaluated as shown in Figure 4a. Absolute choline peak area had the most 


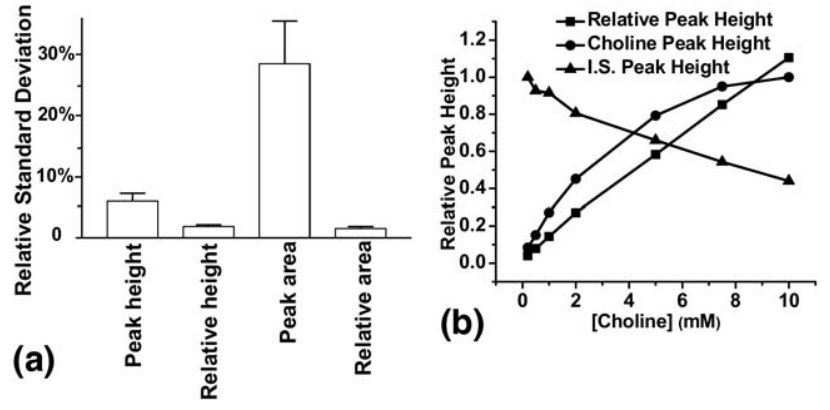

Figure 4. Quantification of AchE hydrolysis. (a) Comparison of relative standard deviation for different methods of quantifying choline signal from RIC traces: peak height is the highest choline ion intensity of all the scans over a sample plug; relative height is the ratio of peak height of choline to that of chlormequat; peak area is the area under all the MS scans of a sample plug; relative area is ratio of the peak area of choline to that of chlormequat. Error bars are \pm 1 standard deviation $(n=7)$. The average RSDs were $5.9 \%, 28.5 \%, 1.9 \%$, and $1.5 \%$ for calculation based on peak height, peak area, relative height, and relative area respectively; (b) calibration curve for choline. Solutions containing $0.9 \mathrm{mM}$ chlormequat and various concentrations of choline (200 $\mu \mathrm{M}$ to 10 $\mathrm{mM}$ ) were infused for ESI-MS analysis. Choline peak intensity increased with its concentration nonlinearly while chlormequat (I.S.) peak intensity decreased with higher choline concentration (normalized peak intensities were used for both choline and chlormequat). Using ratio of the two peak heights (relative peak height) corrected the effect caused by charge competition during ESI so that the ratio increased linearly with choline concentration. The calibration curve based on relative peak height had slope of $0.11 \mathrm{mM}^{-1}$, y-intercept of 0.034 , and $\mathrm{r}^{2}$ of 0.999 .

variability, which was not surprising because the size of sample plugs had $25 \%$ variability. Peak heights were less variable but could sometimes be affected by fluctuation in electrospray stability. Choline peak area and height relative to the internal standard had low variability, and both proved to be equally acceptable for quantification.

Charge competition between choline and internal standard chlormequat during electrospray and its effect on quantification was also evaluated. Choline signal intensity was measured from $200 \mu \mathrm{M}$ to $10 \mathrm{mM}$ with a fixed chlormequat concentration. This concentration range was tested based on the amount of choline formed during the enzyme assay. (Our previous work has suggested that lower concentrations can be used for assays that require it as the segmented flow electrospray method has high sensitivity [17].) As shown in Figure $4 \mathrm{~b}$, choline signal increased with its concentration nonlinearly while chlormequat signal decreased with increasing choline concentration. By using choline signal relative to the internal standard, a linear calibration curve could be obtained, demonstrating that the use of internal standard also helped to correct for charge competition during ESI at different choline concentrations. In principle, an isotope-labeled internal standard could be also used to correct for ionization suppression if necessary.

Figure $3 \mathrm{~b}$ summarizes quantification of the assay screen shown in Figure 3a using peak area ratio for choline and internal standard. Four of the known AchE inhibitors showed reduced choline production as expected. Interestingly, isoproterenol and DMSO also showed some inhibition at this concentration. DMSO increased signal of both choline and chlormequat; however, quantification was not affected since relative signal intensities were used. This result indicates that the assay should be resistant to compounds that have generalized effects on the ESI-MS process.

The reproducibility of the assay can be evaluated using the $Z^{\prime}$-factor [33]. $Z^{\prime}$-factor is defined as $Z^{\prime}=$ $1.0-\left(3.0 \times\left(s_{\text {neg }}+s_{p o s}\right) / R\right.$ where $s_{\text {neg }}$ is the standard deviation of the response of a negative control (no inhibitor) and $s_{\text {pos }}$ in the standard deviation of the response of a positive control (with inhibitor) and $\mathrm{R}$ is the difference in signal between the mean of positive and negative controls. $Z^{\prime}$ over 0.5 is generally considered necessary for HTS. In our experiments, $Z^{\prime}$ values for neostigmine, eserine, malathion and edrophonium were $0.84,0.83,0.87$ and 0.85 respectively. High $Z^{\prime}$ values were the direct result of excellent reproducibility.

\section{AchE Inhibitor Characterization}

Another use of the assay is for rapid determination of dose-response relationships for known inhibitors as illustrated for neostigmine, eserine, malathion, and edrophonium in Figure 5. For this experiment, 10 different concentrations of each inhibitor ranging from 0 $\mathrm{nM}$ to $10 \mathrm{mM}$ were incubated with the assay mixtures for $20 \mathrm{~min}$ at room temperature. The quenched reaction mixtures were analyzed and absolute choline formation was derived from the choline calibration curve. $\mathrm{IC}_{50} \mathrm{~s}$ of eserine, malathion, and edrophonium were calculated to be $63 \pm 13 \mathrm{nM}, 480 \pm 70 \mu \mathrm{M}, 63 \pm 11 \mu \mathrm{M}$, respectively. Neostigmine resulted in two $\mathrm{IC}_{50}$ values, $50 \pm 25 \mu \mathrm{M}$ and $38 \pm 10 \mathrm{nM}$, based on two-site competition fitting. These numbers generally agreed well with previously reported values (eserine 72-109 $\mathrm{nM}$ [34], malathion $370 \mu \mathrm{M}$ [35], edrophonium $5.4 \mu \mathrm{M}$ [36], and neostigmine $11.3 \mathrm{nM}$ [37]; however, direct comparison of these numbers might not be appropriate because the experimental conditions were not identical (e.g., use of surrogate substrates and different AchE in other assays).

\section{Conclusions}

We demonstrated that AchE inhibitors could be analyzed at throughput of $1.5 \mathrm{~s} /$ sample by preparing samples as an array of individual nanoliter plugs segmented by air and rapidly analyzing them using ESI-MS serially. The throughput of analysis compares favorably with flow injection methods. Further improvements in throughput are possible by using smaller sample plugs and higher flow rates. Another advantage of segmented flow analysis relative to flow injection approaches is the low sample volume requirement. 

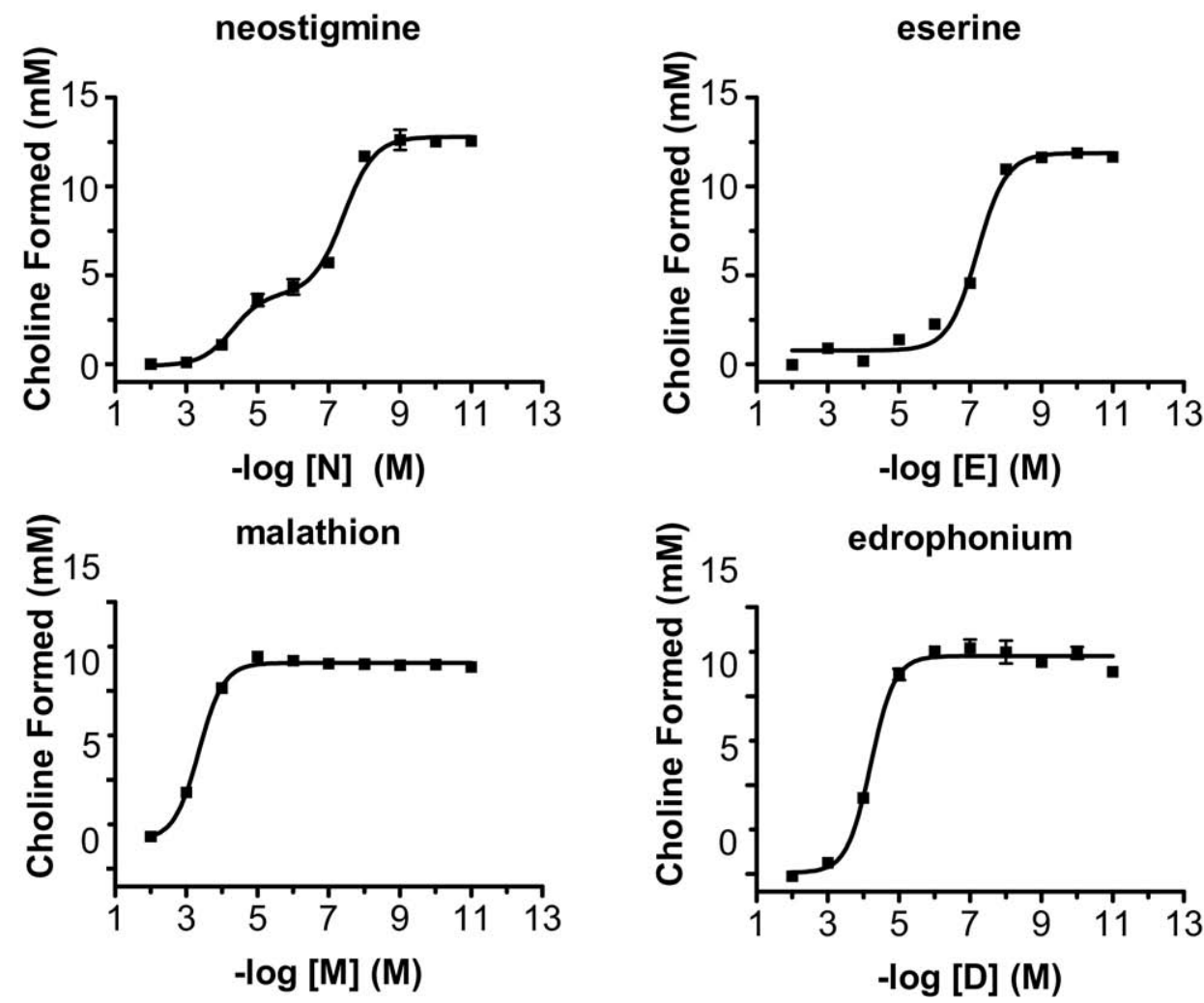

Figure 5. Dose-response curves of four AchE inhibitors. Choline formed when incubated with various inhibitor concentrations were fit to sigmoidal dose-response curves except for neostigmine, which was fit to a two-site competition curve. Error bars are \pm 1 SD $(n=3)$.

Only $10 \mathrm{~nL}$ of sample was consumed in this assay because there is no need to fill and rinse an injection loop. Of course, the total sample used depends on the volume required to collect the $10 \mathrm{~nL}$ sample. In principle, it should be possible to aspirate sample from much lower volume wells than used here.

Although the rate of analysis and sample utilization demonstrated here are compatible with HTS, developing a true HTS system around this approach requires further development. Overall throughput was limited by sample loading in this case; therefore implementation of more rapid or parallel sample loading procedures is necessary. Extending the approach to assays that are not compatible with direct ESI injection will require developing and applying methods of highthroughput sample preparation.

\section{Acknowledgments}

The authors acknowledge support for this work by NSF-CHE 0514638. The ESI source and emitter tips were generously donated by New Objective.

\section{References}

1. Gomez-Hens, A.; Aguilar-Caballos, M. P. Modern Analytical Approaches to High-Throughput Drug Discovery. Trac-Trends Anal. Chem. 2007, 26, 171-182.

2. Hodder, P.; Mull, R.; Cassaday, J.; Berry, K.; Strulovici, B. Miniaturization of Intracellular Calcium Functional Assays to 1536-Well Plate
Format Using a Fluorometric Imaging Plate Reader. J. Biomol. Screen. 2004, 9, 417-426.

3. Miraglia, S.; Swartzman, E. E.; Mellentin-Michelotti, J.; Evangelista, L.; Smith, C.; Gunawan, I.; Lohman, K.; Goldberg, E. M.; Manian, B.; Yuan, P. M. Homogeneous Cell- and Bead-Based Assays for High Throughput Screening Using Fluorometric Microvolume Assay Technology. J. Biomol. Screen. 1999, 4, 193-204.

4. Hodgson, L. New Approaches to In-Cell Detection of Protein Activity: Genetically Encoded Chemiluminescence Probes Pave the Way to Robust HTS Assays. ACS Chem. Biol. 2008, 3, 335-337.

5. Liesener, A.; Karst, U. Monitoring Enzymatic Conversions by Mass Spectrometry: A Critical Review. Anal. Bioanal. Chem. 2005, 382, 14511464 .

6. Zehender, H.; Mayr, L. M. Application of Mass Spectrometry Technologies for the Discovery of Low-Molecular Weight Modulators of Enzymes and Protein-Protein Interactions. Curr. Opin. Chem. Biol. 2007, $11,511-517$.

7. Hofstadler, S. A.; Sannes-Lowery, K. A. Applications of ESI-MS in Drug Discovery: Interrogation of Noncovalent Complexes. Nat. Rev. Drug Discov. 2006, 5, 585-595.

8. Mathur, S.; Hassel, M.; Steiner, F.; Hollemeyer, K.; Hartmann, R. W. Development of a New Approach for Screening Combinatorial Libraries Using MALDI-TOF-MS and HPLC-ESI-MS/MS. J. Biomol. Screen. 2003, 8, 136-148.

9. Gao, J. M.; Cheng, X. H.; Chen, R. D.; Sigal, G. B.; Bruce, J. E.; Schwartz, B. L.; Hofstadler, S. A.; Anderson, G. A.; Smith, R. D.; Whitesides, G. M. Screening Derivatized Peptide Libraries for Tight Binding Inhibitors to Carbonic Anhydrase II by Electrospray Ionization Mass Spectrometry. J. Med. Chem. 1996, 39, 1949-1955.

10. Shiau, A. K.; Massari, M. E.; Ozbal, C. C. Back to Basics: Label-Free Technologies for Small Molecule Screening. Comb. Chem. High Throughput Screen. 2008, 11, 231-237.

11. Van Pelt, C. K.; Zhang, S.; Fung, E.; Chu, I. H.; Liu, T. T.; Li, C. Korfmacher, W. A.; Henion, J. A Fully Automated Nanoelectrospray Tandem Mass Spectrometric Method for Analysis of Caco-2 Samples. Rapid Commun. Mass Spectrom. 2003, 17, 1573-1578.

12. Furman, W. B. Continuous-Flow Analysis. Theory and Practice; CRC press: New York, 1976

13. Snyder, L. R. Continuous-Flow Analysis-Present and Future. Anal. Chim. Acta 1980, 114, 3-18.

14. Song, H.; Chen, D. L.; Ismagilov, R. F. Reactions in Droplets in Microfluidic Channels. Angew. Chem. Int. Ed. 2006, 45, 7336-7356. 
15. Sgro, A. E.; Allen, P. B.; Chiu, D. T. Thermoelectric Manipulation of Aqueous Droplets in Microfluidic Devices. Anal. Chem. 2007, 79, 48454851.

16. Fidalgo, L. M.; Abell, C.; Huck, W. T. S. Surface-Induced Droplet Fusion in Microfluidic Devices. Lab. Chip 2007, 7, 984-986.

17. Pei, J.; Li, Q.; Lee, M. S.; Valaskovic, G. A.; Kennedy, R. T. Analysis of Samples Stored as Individual Plugs in a Capillary by Electrospray Ionization Mass Spectrometry. Anal. Chem. 2009, 81, 6558-6561.

18. Fidalgo, L. M.; Whyte, G.; Ruotolo, B. T.; Benesch, J. L. P.; Stengel, F.; Abell, C.; Robinson, C. V.; Huck, W. T. S. Coupling Microdroplet Microreactors with Mass Spectrometry: Reading the Contents of Single Droplets Online. Angew. Chem. Int. Ed. 2009, 48, 3665-3668.

19. Kelly, R. T.; Page, J. S.; Marginean, I.; Tang, K. Q.; Smith, R. D. Dilution-Free Analysis from Picoliter Droplets by Nano-Electrospray Ionization Mass Spectrometry. Angew. Chem. Int. Ed. 2009, 48, 68326835.

20. Perry, E. Acetylcholine and Alzheimer's Disease. Br. J. Psychiat. 1988, $152,737-740$

21. Greenblatt, H. M.; Dvir, H.; Silman, I.; Sussman, J. L. Acetylcholinesterase-a Multifaceted Target for Structure-Based Drug Design of Anticholinesterase Agents for the Treatment of Alzheimer's Disease. J. Mol. Neurosci. 2003, 20, 369-383.

22. Maidment, I.; Fox, C.; Livingston, G.; Katona, C. Drug Treatment for Alzheimer's Disease: The Way Forward. Int. J. Geriatr. Psychiat. 2006, 21, $6-8$

23. Ellman, G. L.; Courtney, K. D.; Andres, V.; Featherstone, R. M. A New and Rapid Colorimetric Determination of Acetylcholinesterase Activity. Biochem. Pharmacol. 1961, 7, 88-96.

24. Rhee, I. K.; Appels, N.; Luijendijk, T.; Irth, H.; Verpoorte, R. Determining Acetylcholinesterase Inhibitory Activity in Plant Extracts Using a Fluorimetric Flow Assay. Phytochem. Anal. 2003, 14, 145-149.

25. Guardigli, M.; Pasini, P.; Mirasoli, M.; Leoni, A.; Andreani, A.; Roda, A Chemiluminescent High-Throughput Microassay for Evaluation of Acetylcholinesterase Inhibitors. Anal. Chim. Acta 2005, 535, 139-144.

26. Ingkaninan, K.; de Best, C. M.; van der Heijden, R.; Hofte, A. J. P.; Karabatak, B.; Irth, H.; Tjaden, U. R.; van der Greef, J.; Verpoorte, R. High-Performance Liquid Chromatography with on-Line Coupled UV, Mass Spectrometric and Biochemical Detection for Identification of Acetylcholinesterase Inhibitors from Natural Products. J. Chromatogr. A $2000,872,61-73$.
27. Ozbal, C. C.; LaMarr, W. A.; Linton, J. R.; Green, D. F.; Katz, A.; Morrison, T. B.; Brenan, C. J. H. High Throughput Screening Via Mass Spectrometry: A Case Study Using Acetylcholinesterase. Assay Drug Dev. Technol. 2004, 2, 373-381.

28. Hu, F. L.; Zhang, H. Y.; Lin, H. Q.; Deng, C. H.; Zhang, X. M. Enzyme Inhibitor Screening by Electrospray Mass Spectrometry with Immobilized Enzyme on Magnetic Silica Microspheres. J. Am. Soc. Mass Spectrom. 2008, 19, 865-873.

29. Andrisano, V.; Bartolini, M.; Gotti, R.; Cavrini, V.; Felix, G. Determination of Inhibitors' Potency (IC50) by a Direct High-Performance Liquid Chromatographic Method on an Immobilized Acetylcholinesterase Column. J. Chromatogr. B 2001, 753, 375-383.

30. Link, D. R.; Anna, S. L.; Weitz, D. A.; Stone, H. A. Geometrically Mediated Breakup of Drops in Microfluidic Devices. Phys. Rev. Lett. 2004, 92, 054503.

31. Chabert, M.; Dorfman, K. D.; de Cremoux, P.; Roeraade, J.; Viovy, J. L. Automated Microdroplet Platform for Sample Manipulation and Polymerase Chain Reaction. Anal. Chem. 2006, 78, 7722-7728.

32. Vuckovic, D.; Cudjoe, E.; Hein, D.; Pawliszyn, J. Automation of SolidPhase Microextraction in High-Throughput Format and Applications to Drug Analysis. Anal. Chem. 2008, 80, 6870-6880.

33. Zhang, J. H.; Chung, T. D. Y.; Oldenburg, K. R. A Simple Statistical Parameter for Use in Evaluation and Validation of High Throughput Screening Assays. J. Biomol. Screen. 1999, 4, 67-73.

34. Vinutha, B.; Prashanth, D.; Salma, K.; Sreeja, S. L.; Pratiti, D.; Padmaja R.; Radhika, S.; Amit, A.; Venkateshwarlu, K.; Deepak, M. Screening of Selected Indian Medicinal Plants for Acetylcholinesterase Inhibitory Activity. J. Ethnopharmacol. 2007, 109, 359-363.

35. Krstic, D. Z.; Colovic, M.; Kralj, M. B.; Franko, M.; Krinulovic, K.; Trebse, P.; Vasic, V. Inhibition of AchE by Malathion and Some Structurally Similar Compounds. J. Enzyme Inhib. Med. Chem. 2008, 23, 562-573.

36. Alvarez, A.; Alarcon, R.; Opazo, C.; Campos, E. O.; Munoz, F. J.; Calderon, F. H.; Dajas, F.; Gentry, M. K.; Doctor, B. P.; De Mello, F. G.; Inestrosa, N. C. Stable Complexes Involving Acetylcholinesterase and Amyloid- $\beta$ Peptide Change the Biochemical Properties of the Enzyme and Increase the Neurotoxicity of Alzheimer's Fibrils. J. Neurosci. 1998, 18, 3213-3223.

37. Iwanaga, Y.; Kimura, T.; Miyashita, N.; Morikawa, K.; Nagata, O.; Itoh, Z.; Kondo, Y. Characterization of Acetylcholinesterase Inhibition by Itopride. Jpn. J. Pharmacol. 1994, 66, 317-322. 